\title{
Flight Simulator Evaluation of Display Media Devices for Synthetic Vision Concepts
}

\author{
J. J. (Trey) Arthur, III*, Steven P. Williams, Lawrence J. Prinzel, III, Lynda J. Kramer and \\ Randall E. Bailey \\ NASA Langley Research Center, Hampton, VA
}

\begin{abstract}
The Synthetic Vision Systems (SVS) Project of the National Aeronautics and Space Administration's (NASA) Aviation Safety Program (AvSP) is striving to eliminate poor visibility as a causal factor in aircraft accidents as well as enhance operational capabilities of all aircraft. To accomplish these safety and capacity improvements, the SVS concept is designed to provide a clear view of the world around the aircraft through the display of computer-generated imagery derived from an onboard database of terrain, obstacle, and airport information. Display media devices with which to implement SVS technology that have been evaluated so far within the Project include fixed field of view head up displays and head down Primary Flight Displays with pilot-selectable field of view. A simulation experiment was conducted comparing these display devices to a fixed field of view, unlimited field of regard, full color Helmet-Mounted Display system. Subject pilots flew a visual circling maneuver in IMC at a terrain-challenged airport. The data collected for this experiment is compared to past SVS research studies.
\end{abstract}

Keywords: Synthetic Vision, Helmet-Mounted Display, Aviation Safety, Situation Awareness, Enhanced Vision

\section{INTRODUCTION}

In commercial aviation, over $30 \%$ of all fatal accidents worldwide are categorized as Controlled Flight Into Terrain (CFIT), where a mechanically sound and normally functioning airplane is inadvertently flown into the ground, water, or an obstacle, principally due to the lack of outside visual reference and situational awareness ${ }^{1}$. The Synthetic Vision Systems (SVS) project, under NASA's Aviation Safety Program (AvSP), is developing technologies with practical applications that will eliminate low visibility conditions as a causal factor to civil aircraft accidents ${ }^{2,3}$.

\subsection{Synthetic Vision Display Concepts}

A major thrust of the SVS project involves the development and demonstration of affordable, certifiable display configurations which provide intuitive out-the-window terrain and obstacle information, including pathway and guidance information for precision navigation and obstacle/obstruction avoidance, for Commercial and Business aircraft. In addition to forward-fit applications, a path to retrofit this technology into today's transport aircraft fleet is also necessary to achieve the desired safety benefits since $66 \%$ of today's transport aircraft fleet is equipped with only electromechanical cockpit instrumentation.

NASA's SVS concept (Figure 1) provides a real-time, unobscured synthetic view of the world for the pilot. The display is generated by visually rendering an on-board terrain database (with additional airport and obstacle database information as necessary) using precise position and navigation data obtained through GPS (Global Positioning System) data, with augmentation possibly from differential correction sources such as Local Area Augmentation Systems (LAAS) and Wide Area Augmentation Systems (WAAS), as well as blending from on-board Inertial Navigation System (INS) information. Active imaging sensors, real-time hazard information (e.g., weather and wake vortices), and traffic information as provided by Automatic Dependent Surveillance - Broadcast (ADS-B) and Traffic Information Services Broadcast (TIS-B) can additionally enhance this synthetic vision display concept (SVDC). Although the display representation to the pilot is synthetically derived, object detection and integrity monitoring functions are envisioned to ensure sufficient accuracy and reliability for certification.

*Trey.Arthur@nasa.gov, NASA LaRC, 24 W Taylor Street, Hampton, VA 23681, phone 17578646609 


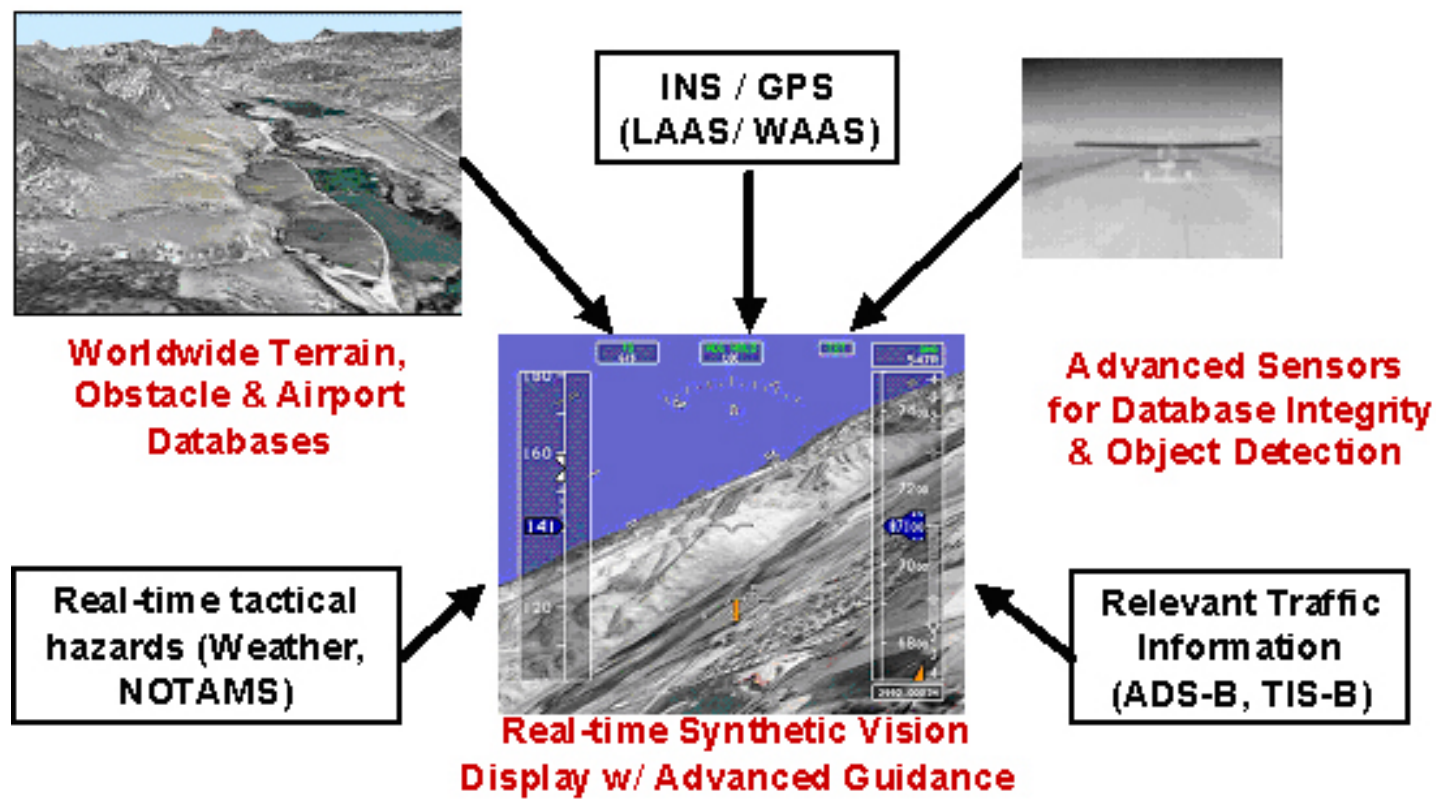

Figure 1: Synthetic Vision System concept.

\subsection{Retrofit Approach}

To date, much of the SVS research has focused on introducing SVS display technology into as many existing aircraft as possible by providing a retrofit approach. This approach employs existing head down display (HDD) capabilities for glass cockpits (cockpits already equipped with raster-capable HDDs) and head-up display (HUD) capabilities for the other aircraft. Two major NASA flight tests have been conducted for assessment and evaluation of the SVS developments. Both flight tests have used the NASA/Langley Research Center (LaRC)-modified Boeing 757-200 jetliner known as the Airborne Research Integrated Experiment System (ARIES). The first flight test was flown Sept-Oct 2000 in nighttime operations at Dallas-Ft. Worth (FAA Identifier: DFW) ${ }^{4}$. The second flight test was flown Aug-Sept 2001 in simulated daylight Instrument Meteorological Conditions (IMC) at Eagle County Regional Airport, CO (FAA Identifier: EGE $)^{5,6}$. One objective of these tests (and precursor simulator studies) was to examine whether SVS technology could be effectively retrofitted into existing size HDDs, such as an ARINC Size A (e.g., B-757-200) Electronic Attitude Direction Indicator (EADI) and an ARINC Size D (e.g., B-777) Primary Flight Display (PFD). A Size "X" head-down display was also tested that may represent the display area available on future aircraft. Each of the display size variations of the SVS HDD concepts evaluated included a pilot-selectable field of view (FOV) feature as a potential mitigating control for the relatively small, fixed display sizes. Two terrain-texturing techniques were also evaluated during the research. One method of terrain texturing, generic texturing, involved the selection of terrain color based on absolute altitude. The other method of terrain texturing, photo-realistic texturing, employed ortho-rectified aerial photographs draped over the elevation model. The results of those studies confirmed that SVS technology, with pilot-selectable FOV, could be effectively incorporated into existing size EADI or PFD displays. Regardless of display size, pilots reported greater situation awareness and obtained lower flight technical error (FTE) while operating with the SVS displays compared to the conventional displays.

A cost-effective retrofit path for SVS in electro-mechanical cockpits may be possible by generation of a synthetic vision image as the raster input source to a stroke-on-raster HUD. This display concept is analogous in many respects to the Enhanced Vision System (EVS) certified on the Gulfstream V, except that the raster image is synthetically-derived rather than being a direct imaging sensor output. Unlike EVS displays, the SVS HUD concept uses a clear sky rather than a sensor image of the sky, so there is no obstruction in that area of the display. Below the horizon, the raster image may obstruct the view of the outside real world (as with an EVS image), particularly if the pilot does not control the 
raster brightness appropriately. In both flight trials, the SVS-HUD concept was, for all intents, just a monochromatic green representation of the full-color, head-down display SVS concept, using an RS-343 video format. Extensive effort was not expended to evaluate optimal graphical light sources or other terrain shading issues. SVS HUD concept evaluations included the same two variations in the method of terrain texturing that were evaluated in the head-down displays. The feasibility of the concept of retrofitting SVS display technology with HUDs was verified, particularly for nighttime operations. Pilots reported greater situation awareness and obtained lower FTE while operating with the SVSHUD concepts compared to the conventional displays. Although promising results were obtained, two significant deficiencies were found in daylight HUD usage: illegible display renditions under some direct sunlight conditions and some reported terrain depiction illusions. For both HDD and HUD applications, no significant performance effects were found between texturing techniques, although most of the pilots preferred the photo-realistic terrain texturing technique to the generic texturing technique.

\subsection{Current SVS research}

Researchers at NASA LaRC conducted an experiment examining the efficacy of different tunnel and guidance concepts for head-down synthetic vision displays. This experiment focused on an SVS head-down PFD and examined four tunnel concepts (box, minimal, dynamic, and pathway) and three guidance symbologies (ball, tadpole, follow-me aircraft). The box tunnel concept (used frequently in past tunnel research) consisted of a series of boxes connected at the corners to form a path within which the pilot flies. The minimal tunnel concept consisted of a series of "crow's feet" which represented the truncated corners of nominally-connected 2-dimensional rectangles spaced at $0.2 \mathrm{~nm}$ increments along the desired path. The crow's feet were linearly decreased in brightness so, by $3.0 \mathrm{~nm}$ from own-ship, the brightness of the bottom crow's feet was reduced to zero. The dynamic tunnel concept had the crow's feet grow as a function of path error to provide the pilots feedback on how well they were flying the defined path. The idea of the dynamic tunnel is to minimize clutter when the pilot is flying on path and to alert them as their path error grows by dynamically lengthening the sides of the tunnel in the direction of the path error. The pathway tunnel concept was a variation of the dynamic tunnel concept in which the floor of the tunnel was presented at all times. For both the dynamic and pathway tunnel concepts, when the pilot left the tunnel, the tunnel would change to a trough that resembled three sides of the box tunnel concept. The tunnel was open on the error side to "invite" the pilot back into the tunnel.

All three guidance concepts (ball, tadpole, follow-me aircraft) were driven by the same modified pursuit guidance laws and only differed in their presentation to the pilot ${ }^{7}$. The guidance symbol was positioned 30 seconds ahead of the ownship nominally on the centerline of the tunnel. Yaw, pitch, and roll attitude changes of the guidance symbol reflected the track and flight path angles of the path at that lead position. The ball symbol was a laterally and vertically integrated guidance cue. The tadpole guidance symbol was the same as the integrated ball guidance with added track change information provided by a vertical line. The idea being that the "tadpole" line gives the pilot lateral anticipation of the guidance symbol. The line pointed straight up if the desired track was constant. As the track changed, it rotated left or right in the direction of the track change to denote desired lateral path. The ghost guidance symbol provided the pilot with visual yaw, pitch, and roll changes. The specific tunnel/guidance concepts tested were: box tunnel/ball guidance, minimum tunnel/ball guidance, pathway tunnel/ball guidance, dynamic tunnel/ball guidance, dynamic tunnel/tadpole guidance, and dynamic tunnel/ghost guidance. The tunnel concepts were evaluated against a baseline concept (no tunnel using the ball guidance symbology). All concepts and the baseline were paired with a navigation display with a Terrain Awareness and Warning System (TAWS).

Results from this SVS head-down experiment indicated that the presence of a tunnel on an SVS primary flight display had a marginal effect on enhancing lateral flight path performance but significant differences were evident for SA and workload. For both SA and workload, the no-tunnel condition (baseline) was rated significantly worse (higher workload and lower SA) than any of the tunnel concepts employed. The dynamic tunnel concept was rated as producing the lowest workload and providing the highest SA among the tunnel concepts evaluated. The choice of guidance symbol had no effect on either path performance or workload but did have a significant effect on SA. The ball was rated significantly lower in SA than either the tadpole or ghost but there were no appreciable differences between these two guidance symbologies. 


\subsection{Current Study}

With limited display area in the cockpit, a helmet-mounted display (HMD) could offer a future display solution for the introduction of SVS technologies. Combined with head tracking, stereoscopic imaging and enhanced and synthetic vision systems, HMDs have the potential to provide the pilot with spatially-integrated information, in an uncluttered format for superior SA and usability compared to today's technology. As the technology progresses, HMDs will eventually be very light weight, high-resolution and reliable displays that can be comfortably worn for extended periods, making them practical in commercial and business aircraft operations, in addition to their current military applications.

The current study used a $4.4 \mathrm{lb}$, full color, monocular (left eye) SXGA (1280x1024 pixel) prototype HMD with a field of view of 41 degrees horizontal by 32.8 degrees vertical. Each subject pilot flew a total of 6 Sparks Visual Arrivals to Runway 16R at the Reno-Tahoe International Airport (FAA Identifer: RNO) in IMC conditions. Three of the data runs were flown using the HMD and three runs were flown with a Size D head-down PFD. Note that the HMD was not worn during the PFD part of the experiment.

This experiment was part of a broader experiment that examined the efficacy of pathway and guidance concepts that may be integrated as part of future synthetic vision head-up displays. In that experiment, two tunnel formats (dynamic, minimum), which were found to be effective in an SVS head-down display application, were evaluated against a baseline condition (no tunnel) during approaches to RNO using the Sparks Visual Arrival to Runway 16R under simulated IMC. The results of this HUD experiment will be compared to the appropriate results of the HMD experiment.

\subsection{Experiment Objectives}

The main purpose of the experiment was to evaluate the potential of HMD for SVS research. The experiment objectives were:

1. Determine pilot usability / acceptability and Situational (Terrain) Awareness provided by a NASA SVDC Helmet-Mounted Display (SVDC-HMD) and assess the potential of the SVDC-HMD as a display solution for SVS concepts.

2. Assess closed-loop performance during manually flown landing approach maneuvers in a terrain-challenged operational environment to determine the effect of SVS on performance, and quantify performance with respect to required navigation performance (RNP) procedures ${ }^{8}$.

\section{METHODOLOGY}

\subsection{Simulation Facility}

The experiment was conducted in the Visual Imaging Simulator for Transport Aircraft Systems (VISTAS) III part task simulator at the NASA LaRC (Figure 2, top). The single pilot fixed-base simulator consists of a 144 degree by 30 degree out the window visual, an HMD, a large field HDD and pilot input controls. The HMD was a prototype system, which consisted of a head tracker, full color, binocular optics with 1280x1024 resolution in each eye. The field of the view of the HMD was 41 degrees horizontal by 32.8 degrees vertical. For this experiment, the out the window scene was used only during training and for the HMD, only the left eye was utilized (due to technical difficulties with the right eye optics).

The pilot controls in the VISTAS III workstation are a left side arm controller, left/right throttle controls, rudder pedals, left/right toe brakes and a voice recognition Personal Computer (PC) for display-related pilot inputs. Voice recognition was used to control the SVS FOV for the HDD. The FOV of the HMD was fixed but the field of regard was slaved to the pilot's head position via head tracking. The head down display was a Size D PFD. There was no Navigational Display (ND) used in this experiment.

Earlier in the day, the same subjects participated in a separate SVS experiment. This separate HUD experiment was conducted in the Integration Flight Deck (IFD) simulation facility (Figure 2, bottom) at NASA LaRC. The IFD emulates LaRC's ARIES Boeing B-757-200 research aircraft's cockpit and provides researchers with a full-mission simulator capability. The simulation cockpit is populated with flight instrumentation, including the overhead subsystem panels, to replicate the B-757 and uses the same pilot controls as ARIES. It also employs a large, collimated field of view display 
system for the out-the-window visuals. For the HUD experiment, the out the window visuals were utilized to emulate IMC conditions. The aircraft broke out of the clouds at approximately 500 feet Above Ground Level (AGL).

Both experiments involved tunnel (pathway in the sky) guidance on an SVS display. In addition, both experiments used the same visual Sparks 16R approach path to the RNO.

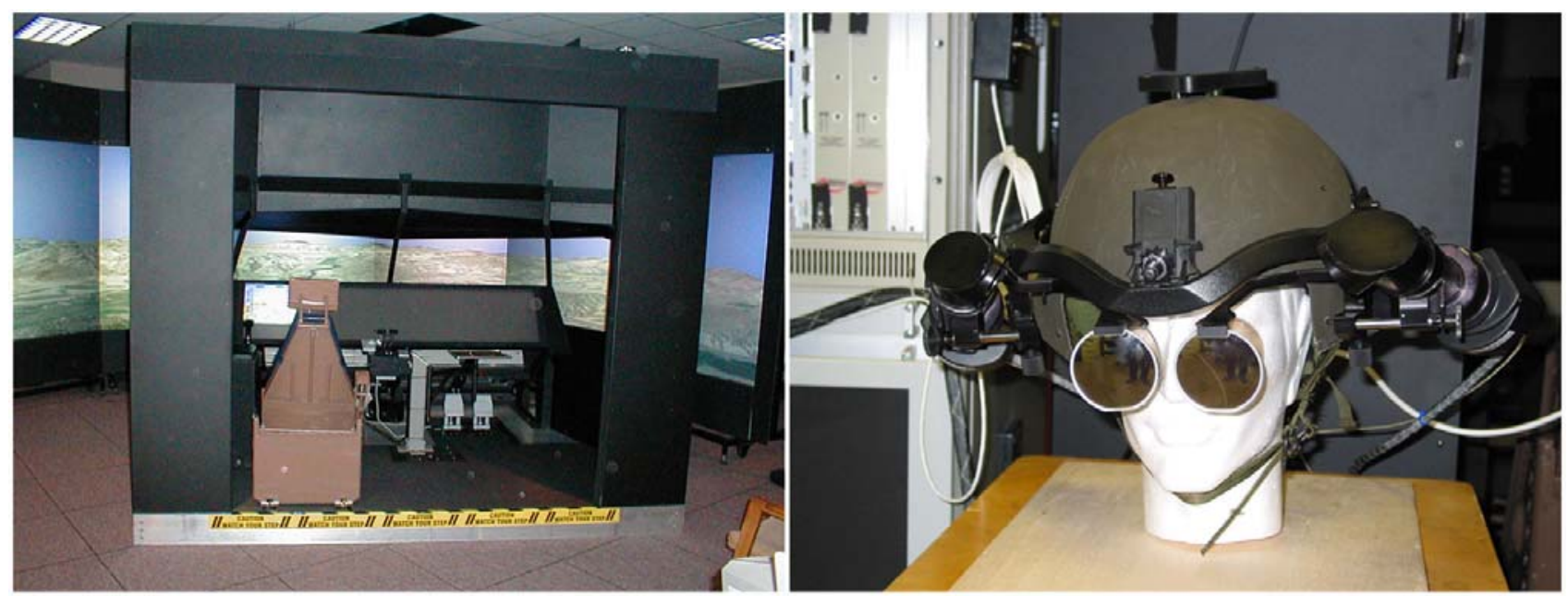

VISTAS III fixed based pilot in the loop workstation and full color prototype HMD.

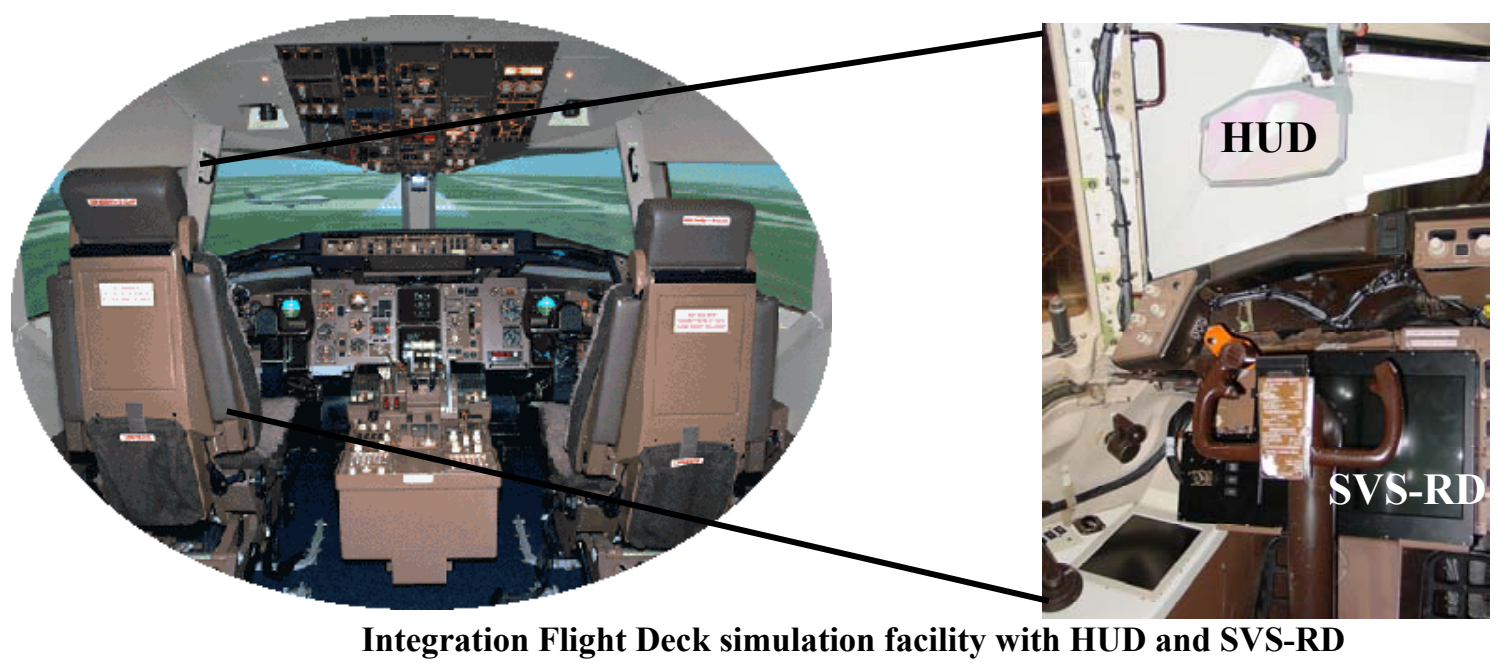

Figure 2: VISTAS III and IFD simulation facilities.

\subsection{Test Subjects}

Eight pilots, representing four airlines and a major transport aircraft manufacturer, participated in the experiment. All participants had HUD experience and all airline pilots had current commercial licenses. The subjects had an average of 683.7 hours of HUD flying experience and an average of 9.7 years and 12.2 years of commercial and military flying experience, respectively. The subjects were given a 30-minute briefing to explain the SVS display concepts and the expected subject task. After the briefing, a 30-minute training session was conducted to familiarize the subjects with the aircraft model, controls and HMD. Six data collection runs lasting approximately 45 minutes were conducted immediately following the training session. The total time for this HMD experiment was approximately 2 hours. 


\subsection{Terrain Texture}

The SVS terrain was displayed in a hybrid textured format. This hybrid texture was created from monochromatic photographs of the RNO area merged with elevation-based color-coded terrain (Figure 3). The process involved coloring the actual aerial photographs of the RNO area based on altitude and draping those elevation-colored photos on top of the digital elevation model of the terrain; thus, creating the hybrid textured terrain. The photographs for the hybrid texture were nested such that high-resolution photographs (1 meter per pixel) were used in close proximity to the RNO airfield with the majority of the database using 4 meters per pixel resolution photographs.

The elevation color-coding used a green color for the field elevation of RNO airport changing toward shades of brown for higher elevations. For this database, dark brown represented altitudes close to field elevation while light browns represented higher elevations. The shading scheme consisted of 14 elevation color bands that were 300 meters in size.

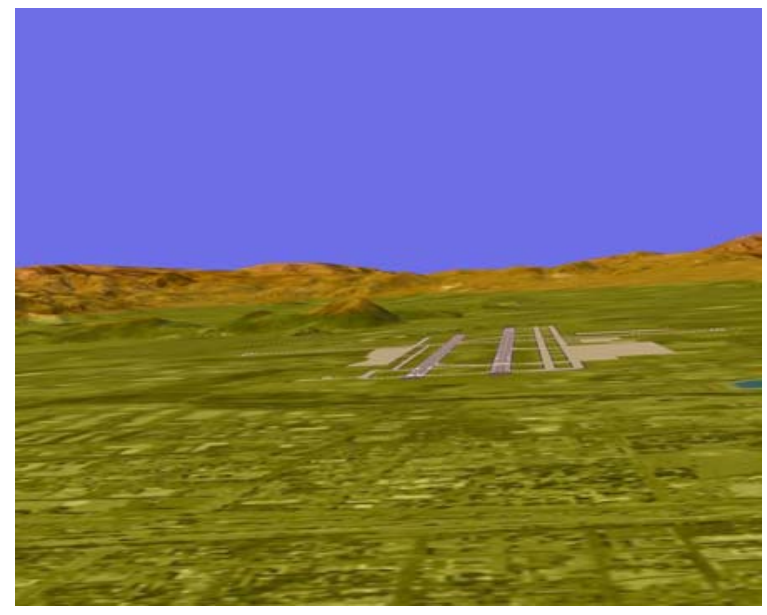

Figure 3: Hybrid textured terrain database of RNO area.

\subsection{Evaluation Tasks}

\section{EXPERIMENTAL TEST}

The test subjects flew a visual approach to RNO runway 16R in IMC (Figure 4). The simulated aircraft was a Boeing 757 flying at 138 knots, indicated airspeed, with no turbulence and no wind. Auto-throttles were enabled, flaps were set to 30 degrees and the landing gear was down. At $200 \mathrm{ft}$ AGL, the data run was terminated. For all data runs, the tunnel and guidance symbology consisted of a dynamic "crow's feet" tunnel with a "tadpole" integrated guidance cue. The approach task took approximately 6 minutes to fly. In addition to the nominal visual arrival approaches, one of the three replicates of the approach task was flown with a simulated ATC call to fly direct to the MCRAN waypoint. For the ATC data run, the MCRAN waypoint was presented on the PFD and HMD as a 3-D waypoint star (Figure 5, on right panel) at the ATC-requested altitude. Once the ATC call was made, the subjects flew outside the tunnel and directly to MCRAN with an ending altitude of 6200 feet (Figure 4). Since there was no ND for either display concept, the star provided a visual aim point for heading and desired altitude. Once arriving at MCRAN, pilots were given another ATC call that they were clear to land, and the pilots' task was to reenter the tunnel and continue the final approach.

The data runs did not start with the subjects inside the tunnel. The start of each run was randomly varied over three conditions: 1) 1000 feet high, 2) 1000 feet right and 3) 500 feet low and 500 feet to the left. The purpose of starting the data runs outside the tunnel was to get subjective feedback as to the task of re-entering the tunnel. As discussed earlier, when outside the tunnel, the rendering of tunnel is fully connected (except for the side of the tunnel the aircraft is closest). Once inside the tunnel, the rendering of the tunnel changed to the dynamic "crow's feet" concept. 


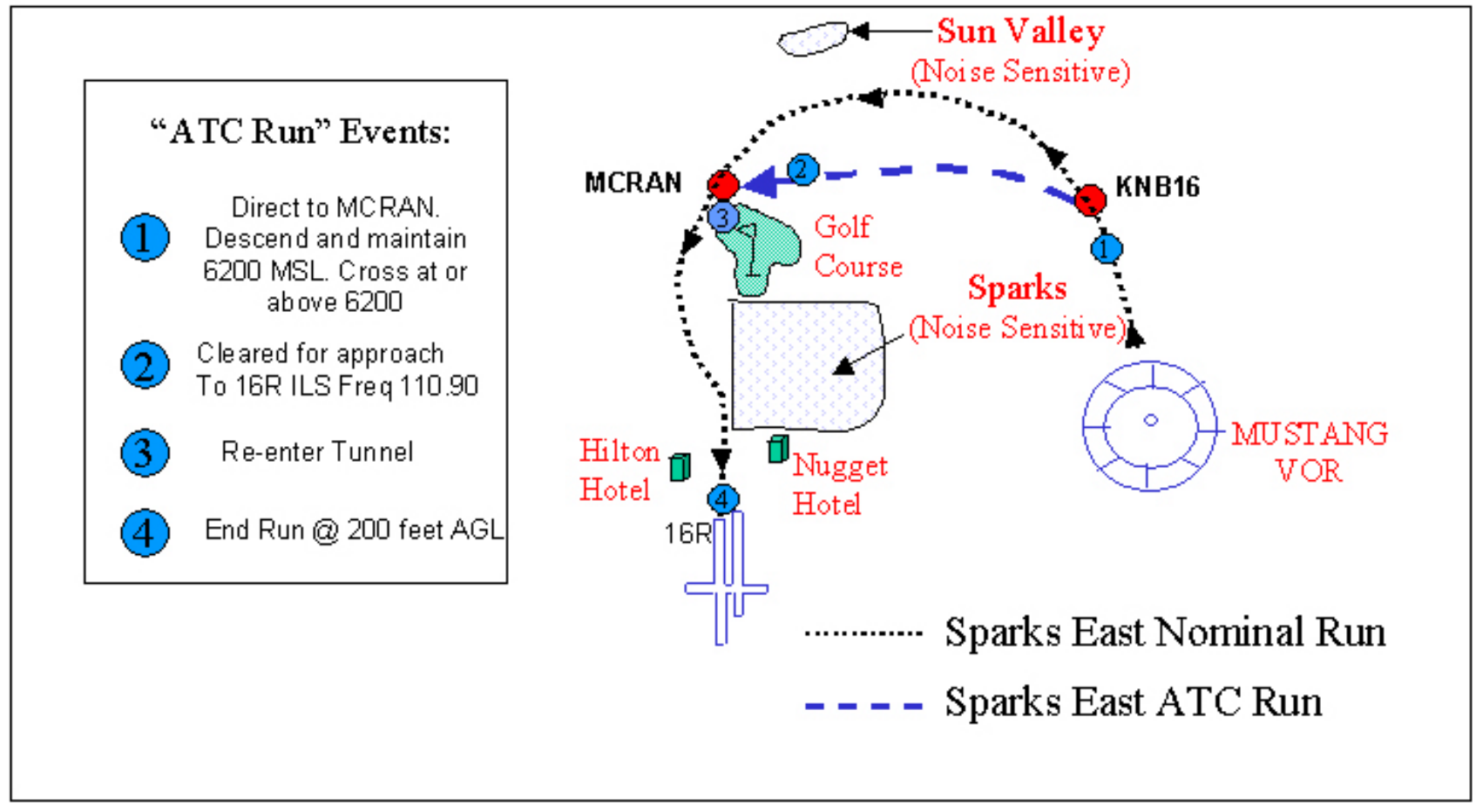

Figure 4: Map view of the visual approach task flown by the subject pilot.
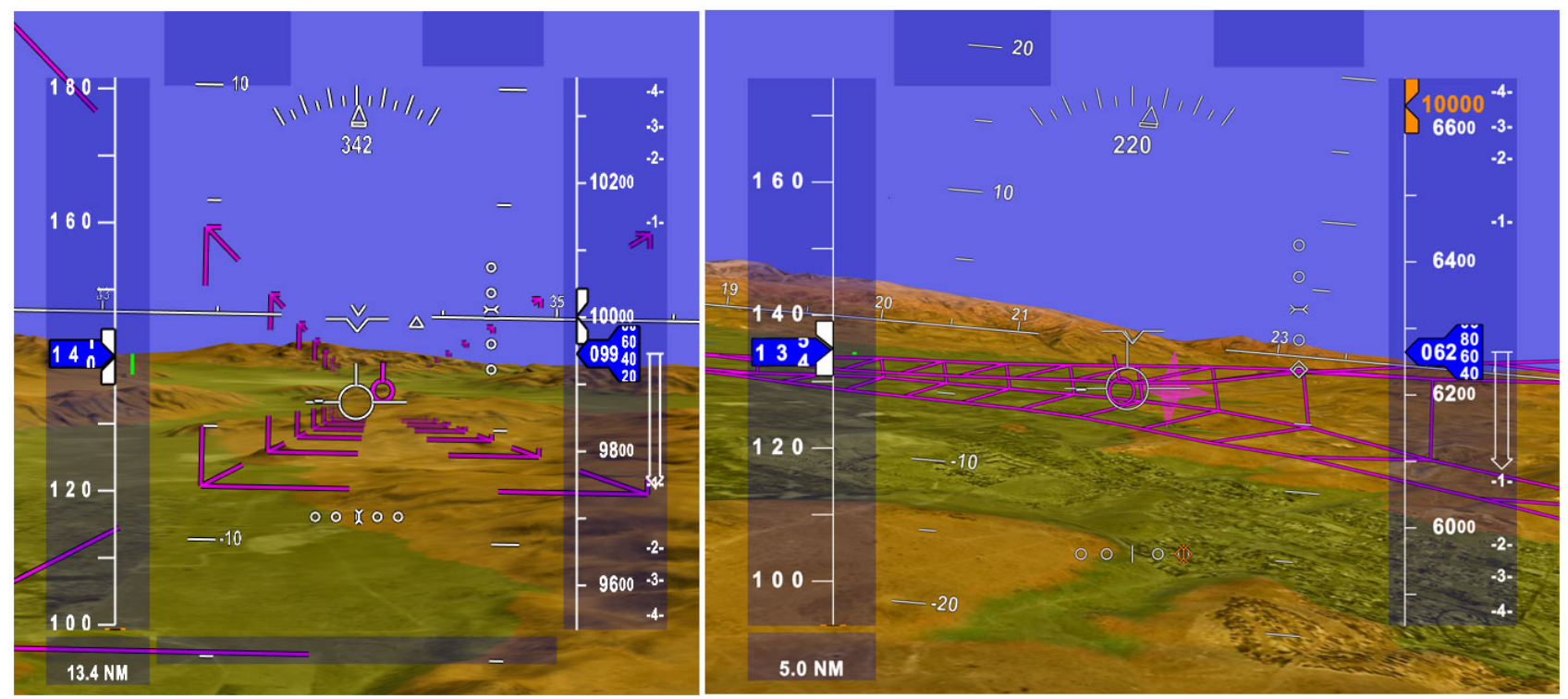

Figure 5: SVS display concept shown on the PFD display and the head tracked color HMD. Left display shows nominal approach task. Right display shows the ATC direct to MCRAN (shown as a star). 


\subsection{Display Conditions}

The subjects flew 3 replications of the same SVS (hybrid terrain with dynamic tunnel and tadpole guidance) concept for each display type. The two display types were a head down Size D PFD and a monocular, color, head-tracked HMD. For one of the three replicates, the approach task was flown with a simulated ATC call to direct to the MCRAN waypoint (Figure 4; Figure 5, right panel).

The experimental run matrix is shown in Table 1.

Table 1: Experiment Run Matrix

\begin{tabular}{|c|c|c|}
\hline Repetitions per pilot & Nominal approach & ATC-shortened approach \\
\hline SVS-PFD (Size D) & 2 & 1 \\
\hline SVDC-HMD & 2 & 1 \\
\hline
\end{tabular}

\section{RESULTS}

\subsection{Flight Path Tracking Results}

Root-mean-square (RMS) calculations of lateral and vertical path error were used as the measures for flight path performance. In addition, a bin analysis of FTE was computed to determine what level of RNP criteria could be achieved while hand-flying an approach. These analyses were all conducted from the full factorial within subject design, and as expected for this precision control task, the main effect of pilot variability was always highly significant for all measures. Only the statistically significant results of the other factors are discussed.

\subsubsection{RNP Results}

Since the start of every run began outside the tunnel (i.e., off path), RNP was not computed until the subjects were established within the tunnel (i.e., on the desired path). Two methods were used for the starting point for the RNP data analysis in an attempt to minimize the influence of the offset initial condition from the results: 1) when 300 foot lateral error and a 175 foot vertical error was reached (i.e., within the tunnel), or, 2) past the 50 second mark from the beginning of the data run. The 50 second time point (Method 2) was always beyond the starting point for Method 1; thus, Method 2 should better represent "steady-state" flight path tracking capabilities. Also note that none of the data runs with ATC calls were included in the RNP analysis.

\subsubsection{RNP Lateral Navigation Analyses}

Lateral path FTE histograms were computed for the two display types (head down PFD or HMD). The path steering error component of the RNP calculation includes both FTE and display error. For this analysis, it was assumed that display error was negligible, so FTE was the only component of path steering error. It was also assumed that the other two components (path definition error and position estimation error) of the RNP calculation would be equivalent across the display concepts evaluated. ${ }^{8,9}$ The data shows no statistically significant differences between display concepts; both display types yielded a lateral FTE navigational accuracy of $0.05 \mathrm{nmi}$ at least $95 \%$ of the time (Figure 6).

\subsubsection{RNP Vertical Navigation Analyses}

Vertical path FTE histograms were computed for the two display types (Size D HDD or HMD). The vertical path steering error component of the VNAV performance calculation includes both FTE and display error. For this analysis, it was assumed that display error was negligible so FTE was the only component of vertical path steering error. It was also assumed that the other three components (altimetry system error, vertical path definition error, and horizontal coupling error) of the VNAV performance calculation would be equivalent across the display concepts evaluated. In addition, it was assumed that the pilot was flying a specified vertical profile so that the required vertical navigation performance accuracy was 210 feet. $^{8}$

Both the PFD and HMD yielded a vertical FTE navigational accuracy of 210 feet at least $99.7 \%$ of the time (Figure 6). As such, based on the vertical path FTE distributions, both display concepts (PFD or HMD) would enable RNP-type operations along a specified vertical profile of 210 feet. 
Comparing these results to previous research results, both the lateral and vertical RNP performance measures are consistent with SVS enhanced displays9. In particular, the companion HUD experiment conducted earlier in the day showed that the subjects achieved similar performance with the same tunnel/guidance symbology. (However, when no tunnel was presented on the SVS-HUD, the vertical RNP requirements were not obtained.)
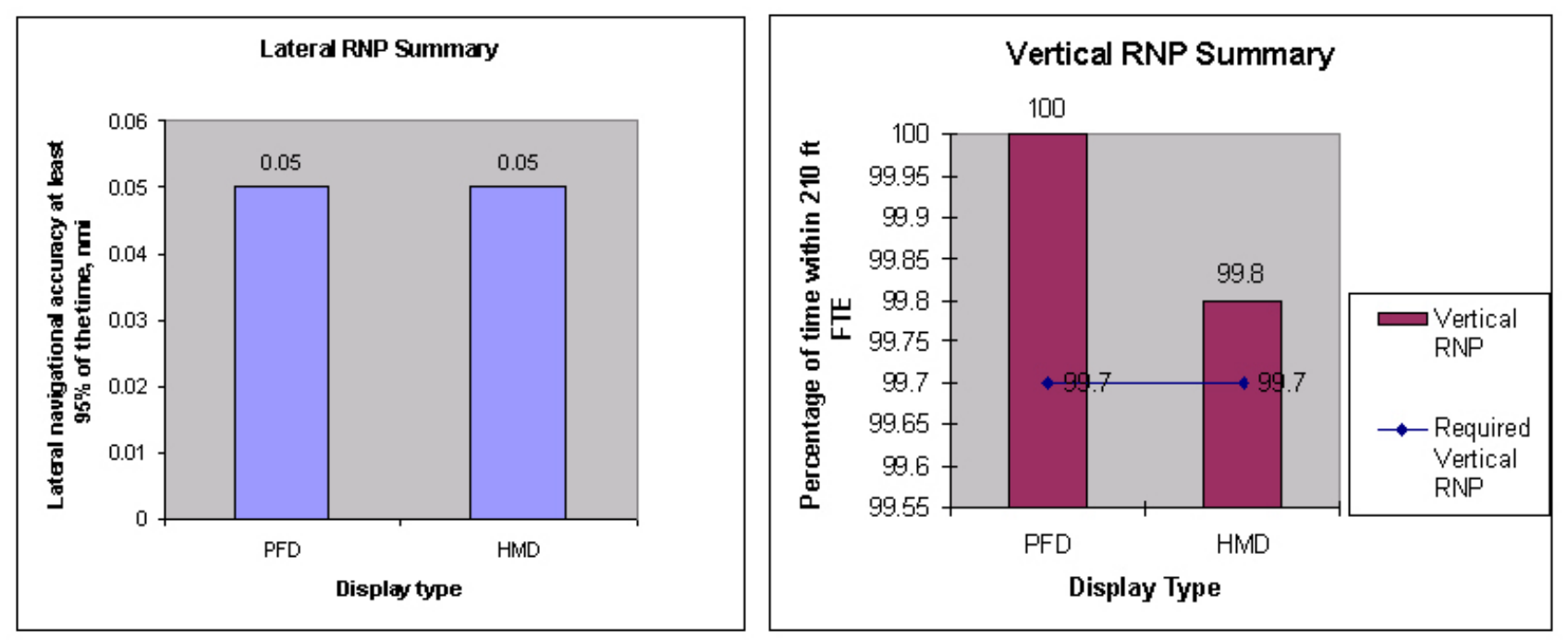

Figure 6: RNP summary for non ATC Run

\subsection{Subjective Results}

After each run, pilots were administered a run questionnaire consisting of the Air Force Flight Technical Center (AFFTC) Revised Workload Estimation Scale ${ }^{10}$ (Figure 7, left panel), Situation Awareness Rating Technique (SART) ${ }^{11}$ (Figure 7, right panel), and six Likert-type (7-point) questions specific to tunnel and guidance symbology evaluation. After data collection was completed, pilots were administered a Subjective Workload Dominance (SWORD) ${ }^{11}$ test. The pilots also participated in a semi-structured interview to elicit comments on the display concepts they had evaluated.

\subsubsection{AFFTC Workload estimate}

The AFFTC workload rating data for each display type (PFD or HMD) was analyzed. After collapsing across the 8 subjects and the three runs for each, the HMD had a mean score of 4.2 (out of 7.0) and the PFD had a score of 3.3/7.0. The subjects rated the task of flying the SVS-PFD display as having "moderate activity" that is "easily managed". The task on the HMD was rated as "busy-challenging" but "manageable". When comparing to the HUD data from the first part of the experiment, subjects rated the HUD similar to the PFD as "moderate activity" that was easily managed.

\subsubsection{SART Results}

The SART measures the pilot's knowledge in three areas: 1) demands on attentional resources, 2) supply of attentional resources, and 3) understanding of the situation. The response to the three SART statements were used to create a single SA score based on the formula that SA $=($ Understanding - (Demand - Supply $)$ ). After collapsing across the 8 subjects and the three runs for each, the SART SA scores were significantly better for the PFD (mean=7.5) than the HMD (mean=4.5). The SVS-PFD was rated as providing more SA. 


\begin{tabular}{||c|l|}
\hline 1 & \multicolumn{1}{c|}{ Workload Es timate } \\
\hline 1 & $\begin{array}{l}\text { Nothing To Do; } \\
\text { No System Demands }\end{array}$ \\
\hline 2 & $\begin{array}{l}\text { Light Activity; } \\
\text { Minimum Demands }\end{array}$ \\
\hline 3 & $\begin{array}{l}\text { Moderate Activity - Easily Managed; } \\
\text { Considerable Spare Time }\end{array}$ \\
\hline 4 & $\begin{array}{l}\text { Busy - Challenging but Manageable; } \\
\text { Adequate Time Available }\end{array}$ \\
\hline 5 & $\begin{array}{l}\text { Very Busy - Dernanding To Manage; } \\
\text { Adequate Time Available }\end{array}$ \\
\hline 6 & $\begin{array}{l}\text { E xtremely Busy - Very Difficult; } \\
\text { Non-E ssertial Tasks Postponed }\end{array}$ \\
\hline 7 & $\begin{array}{l}\text { Overloaded - System Unmanageable; } \\
\text { Essential Tasks Undone; Unsafe }\end{array}$ \\
\hline
\end{tabular}

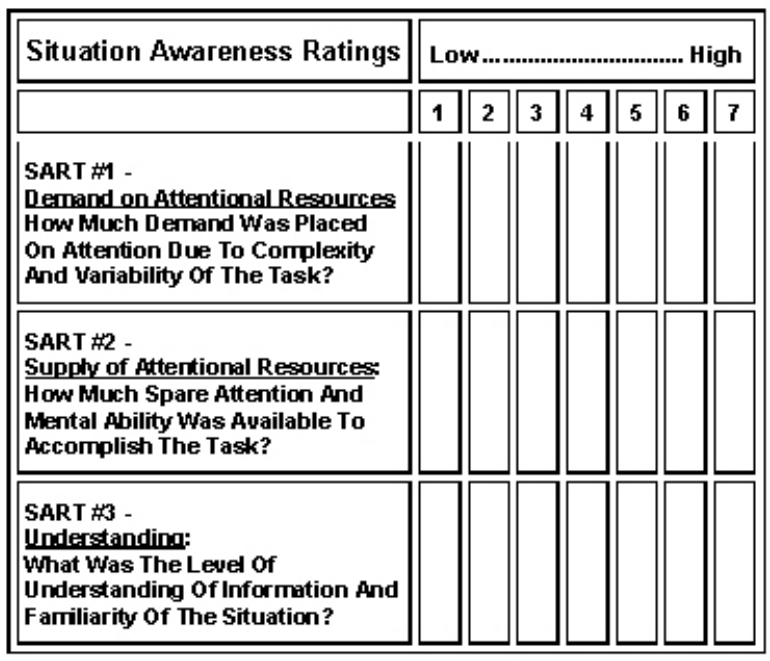

Figure 7: AFFTC Workload and SART scales.

\subsubsection{SWORD Results}

Subjects were asked to rate their workload using the SWORD test for each display type. SWORD was used to obtain the workload on the three different display types (HUD from the earlier experiment, PFD and HMD). Display type was found to be highly significant ( $\mathrm{p}<.001)$. Post hoc tests showed 2 distinct subsets: 1) HUD, PFD and 2) HMD with the lowest workload attributed to the HUD (but which could not be statistically discriminated from the PFD). However, the HMD had significantly more workload than the HUD or PFD.

\subsubsection{Post run Questionnaire Results}

Six post-run questions were asked of each pilot to help assess specific subjects of interest.

1. As I performed the task, my awareness of where I was in the tunnel was

2. As I performed the task, my awareness of upcoming turns using the tunnel was

3. As I performed the task, my level of flight path control and performance was

4. As I performed the task, my ability to intercept the path and re-enter the tunnel was

5. As I performed the task, my ability to anticipate flight path changes using the guidance symbol was

6. As I performed the task, my awareness of terrain features and obstacles was

Subjects scored each statement with a value of 1 through 7 with 1 meaning low and 7 meaning high. An ANOVA was performed on the mean rating for each of those post-run questions with display type (PFD and HMD) and pilot as the independent variables. Only Question 3 ("As I performed the task, my level of flight path control and performance was

") of the six post-run questions had significant differences among the two display types. Subjects felt their flight path control and performance was lower (worse) with the HMD (mean=3.6) than with the PFD (mean=5.2).

\subsubsection{Semi-structured Interview Results}

At the end of all data collection, subjects were asked questions using a semi-structured interview format. Subjects were asked to rank order the display concepts (PFD or HMD) in terms of flight path awareness and performance. Six of the eight subjects rated the PFD as being superior to the HMD. Subjects were then asked to quantify the relative difference between the two display concepts on a scale of 1 to 7 with 1 being minimal difference, 4 being a significant difference and 7 being completely different. Of the six subjects who rated the PFD higher, the mean score was 4.6/7.0, which equates to a significant difference. Pilots believed their flight path awareness and performance was significantly better with the PFD. However, the data analysis showed no significant differences in lateral and vertical path error between the PFD and HMD. From the AFFTC workload scale and the run questionnaire, pilot workload was significantly higher for the HMD than for the PFD. Given the higher workload of the HMD, subjects felt that their performance had suffered as well. Two of the eight subjects felt their performance improved with the HMD over the PFD. Of the two pilots, they believed they had better path awareness and that they performed significantly better with the HMD with a mean rating of 4.5/7.0. 
Subjects were asked to assess their workload when they left the tunnel and re-entered the tunnel during the ATC vectoring maneuver, on a scale of 1 to 7 with 1 being very low workload, 4 being moderate workload and 7 being very high workload. An ANOVA found that the display type (PFD, HMD) was not a significant factor in this workload rating for ATC scenario. The overall mean rating of 3.4 for both display concepts equates to slightly less than moderate workload.

Subjects were asked to provide their opinion on the advantages and disadvantages of the HMD and PFD. Pilots felt the field of regard of the HMD was an advantage to enhance SA. Subjects found the HMD to be especially helpful during the ATC run because they could look at the MCRAN waypoint when it was 90 degrees off aircraft heading. The disadvantage of the HMD was weight/bulkiness and the single eye monocular display. Pilots felt the advantages of the PFD were it's familiar location, variable FOV, and image stability (i.e., it lacked any "jitter" from head tracking). The disadvantage of the PFD was that it was head down and the pilot was not looking outside like when using the HUD and HMD.

Finally, the subjects were asked to provide an overall rank order of the synthetic vision display type they would prefer to use to make the Sparks RNO approach in IMC, including the companion SVS-HUD experiment flown in the morning. Subjects were instructed to ignore simulator differences in their decision (e.g., they were asked not to bias their judgment of the HMD because they did not prefer the side-arm controller of VISTAS III). The overall rank order of the display concepts (Figure 8) was: 1) HUD, 2) PFD and 3) HMD.

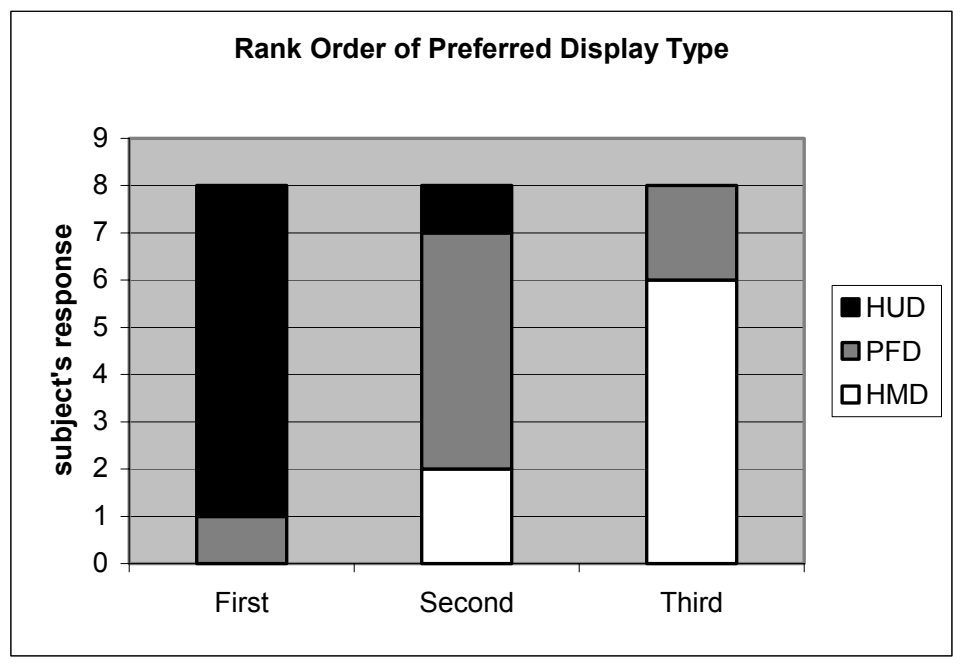

Figure 8: Subjects rank ordering of the three display concepts: HUD, PFD and HMD.

\section{DISCUSSION}

The results from this initial SVS enhanced HMD experiment showed the promise and pitfalls of introducing this new technology into commercial airline-type operations. The "promise" of the SVS-HMD is that it has unlimited field of regard (unlike a fixed FOV HUD) and it has an "out-the-window" look capability (unlike the head down Size D PFD). The results of this experiment showed that an SVS-HMD did not significantly affect flight technical error. Further, all evaluation pilots recognized the potential for increased situation awareness using unlimited field-of-regard display media. In particular, pilots with significant military background recognized the benefits of an SVS-HMD.

However, the data from this experiment also showed that, as currently implemented, the pilot workload and situation awareness were degraded on the HMD compared to a PFD. The pilot commentary highlighted that implementation details, not the concept, were the primary determinates of the usability and acceptability of the SVS-HMD. In particular, the HMD weight and monocular optics were key factors. SVS-HMD implementations will not be accepted without 
significant reductions in size and weight compared to the tested version. The optics qualities must approach eye limiting resolution and image stability (i.e., minimal system latency and head-tracker noise/jitter). Binocular and stereo presentations are being investigated as well to improve the implementation.

Additional concerns for future research will be the implementation of virtual HUDs and PFDs, coupled with "offboresight" symbology since the evaluation pilots emphasized the importance of keeping spatial-integrated symbology.

\section{CONCLUSIONS}

Eight subject flew a visual arrival to RNO using a SVS enhanced Size D head down PFD and a full color head tracked HMD. Both displays were enhanced with SVS including a dynamic tunnel (pathway in the sky) with a "tadpole" guidance cue. With both display concepts, subjects' path performance was comparable to other SVS experiments. However, the HMD was rated to have a higher workload compared to the PFD and HUD. For both display concepts, lateral RNP was within $0.05 \mathrm{nmi} 95 \%$ of the time and the vertical RNP was within required limits. However, subjects rated the workload higher with the HMD compared to the PFD and HUD. The higher workload of the HMD is believed to be attributed to the effort needed to maintain a stable head position to keep the display symbology from jittering and the fact that the helmet was monocular. Future research in SVS enhanced HMDs will include binocular, stereoscopic presentation of display symbology and synthetic terrain.

\section{REFERENCES}

1. Boeing (1998). Statistical summary of commercial jet aircraft accidents, Worldwide Operations, 1959-1997. Seattle, WA: Airplane Safety Engineering, Boeing Commercial Airplane Group.

2. Arthur, J.J., Prinzel, L.J., Kramer, L.J., Bailey, R.E., and Parrish, R.V. (2003). CFIT Prevention Using Synthetic Vision. SPIE. In Proceedings of SPIE, Enhanced and Synthetic Vision 2003, Editor: Jacuqes G. Verly, Volume 5018 paper 16 Apr.

3. Baize, D.G. and Allen, C.L., "Synthetic Vision Systems Project Plan, Version IIId," NASA Langley Research Center, 5 Nov 2001.

4. Glaab, L.J., Kramer, L.J., Arthur, T., \& Barry, J.S. (2003). Flight test comparison of synthetic vision display concepts at Dallas/Fort Worth International airport. NASA Langley Research Center: NASA Technical Paper TP2003-212177.

5. Bailey, R.E., Parrish, R.V., Arthur III, J.J., and Norman, R.M., "Flight Test Evaluation of Tactical Synthetic Vision Display Concepts in a Terrain-Challenged Operating Environment" In Proceedings of SPIE, Enhanced and Synthetic Vision 2002, Editor: Jacuqes G. Verly, Volume 4713, pp. 178-189, Apr 2002.

6. Prinzel, L.J., Kramer, L.J., Bailey, R., Hughes, M., \& Comstock, R., "NASA Eagle-Vail Synthetic Vision Flight Test," Proceedings of the Human Factors and Ergonomics Society 46th Annual Meeting, 46.135-139, 2002

7. Merrick, V.K., Jeske, J.A., "Flight Path Synthesis and HUD Scaling for V/STOL Terminal Area Operations," NASA TM-110348, April 1995.

8. RTCA (2000). Minimum Aviation System Performance Standards: Required Navigation Performance for Area Navigation (RTCA DO-236A). Washington, D.C.: RTCA, Incorporated.

9. Kramer, L.J., Prinzel, L.J., Bailey, R.E., \& Arthur, J.J. (2003). Synthetic vision enhances situation awareness and RNP capabilities for terrain-challenged approaches. Proceedings of the American Institute of Aeronautics and Astronautics Third Aviation Technology, Integration, and Operations Technical Forum, AIAA 2003-6814, 1-11.

10. Ames, Lawrence L. \& George, Edward J. (1993). Revision and verification of a seven-point workload estimation scale. Air Force Flight Technical Center: AFFTC-TIM-93-01.

11. AIAA (1993). Guide to Human Performance Measurement. Washington D.C.: American Institute of Aeronautics and Astronautics. 